\title{
Voltage Profile Correction in Distribution Grids Combining Single- and Two-level Controllers
}

\author{
Hamid Soleimani Bidgoli \\ Dept. of Elec. Eng. and Comp. Science \\ University of Liège, Belgium \\ h.soleimani@ulg.ac.be
}

\author{
Thierry Van Cutsem \\ Fund for Scientific Research (FNRS) \\ University of Liège, Belgium \\ t.vancutsem@ulg.ac.be
}

\begin{abstract}
A real-time control scheme is proposed to correct undesired voltages in distribution networks by adjusting the reactive powers produced by Dispersed Generation Units (DGUs). This is performed at two levels: locally, in DGU controllers, and centrally by a supervisory controller acting on some of the DGUs only. The centralized, discrete controller solves a multitime-step constrained optimization inspired of Model Predictive Control. Unacceptable voltage deviations are partially but rapidly corrected by the local controls. Further but slower corrections are applied by the upper level, taking advantage of the coordination between DGUs. This hybrid control is of practical interest where the deployment of the upper level control is not feasible or affordable over all DGUs. The proposed method effectiveness is demonstrated on a 75-bus test system.

Index Terms-Active distribution networks, real-time voltage control, decentralized control, coordinated control, model predictive control
\end{abstract}

\section{INTRODUCTION}

The ever increasing integration of renewable energy sources is expected to create new technical problems in mediumvoltage distribution systems, for instant over- or undervoltages. To address this issue, and owing to the progress in communication systems, the DGUs can be used as control means. Over the recent years, a considerable research effort has been devoted to their real-time control, as reported for instance in the survey [1].

Various control schemes have been proposed to this purpose. The most widely contemplated, and simplest approach is local (or decentralized) control. It is already present in various grid codes [2]. An alternative with more information exchange among controllers is the agent-based architecture, as proposed for instance in [3] to control the DGUs of a low-voltage grid. The work has been complemented by considering different local reactive power characteristics and comparing the corresponding system behaviours.

Centralized, coordinated control of DGUs, relying on the concept of Model Predictive Control (MPC), was proposed in Ref. [4]. Using a sensitivity model, the controller coordinates the power outputs of DGUs to smoothly bring the unacceptable voltages within specified limits.

Research supported by Public Service of Wallonia, Department of Energy and Sustainable Building, within the framework of the GREDOR project.

The valuable discussions with Dr. Mevludin Glavic are gratefully acknowledged.
A combination of both local and centralized control has been proposed by the authors in Ref. [5]. The local level provides a fast response to a voltage disturbance, reducing its impact and enhancing voltage quality. The centralized control uses measurements collected throughout the network to bring the voltages inside tighter limits and balance the various DGU contributions. A combined scheme was also investigated in [6], with the fast response of the local controls. The centralized control, using the power injection predictions, adjusts regularly the droop parameters of local ones, ensuring that the voltages are kept within their limits in the forecast time interval. Reference [7] proposed a two-stage strategy for distributed energy storage management. In day-ahead, the optimized battery charge/discharge schedules are calculated centrally. They are communicated to local controls for further short-term adjustments, when approaching real time. Using a dynamic model of the system, Ref. [8] suggested a multilayer control structure. At the upper level, a static OPF computes reference values of reactive powers. The latter are communicated to the next layer, an MPC-based centralized controller, which handles the operation constraints.

This paper further extends the work in [5] by considering a hybrid control structure where, at the lower level, the DGUs are all equipped with fast-responding local controllers and, at the upper level, only a subset of the DGUs are centrally controlled. This hybrid configuration is of practical interest in so far as the deployment of the upper level coordinated control may not be feasible or affordable over all DGUs. For instance, it may not be justified to centrally control DGUs of small nominal powers, or those based on an older technology. One of the purposes of this paper is to demonstrate the performance of the upper level having limited controllability of the DGUs.

\section{THE HYBRID CONTROL ARCHITECTURE}

Different control architectures can be considered in a distribution system taking into account practical needs, technical limitations of the DGUs, and regulatory policies.

Local control is performed by the DGU controllers based on local voltage and (active/reactive) power measurements only. The latter are collected without communication delay. Thus, a fast reaction is obtained while no communication infrastructure is required. 


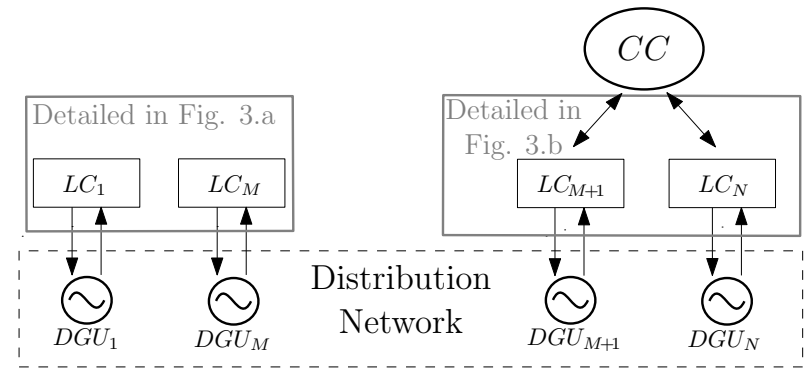

Fig. 1. Hybrid control architecture

A centralized controller, on the other hand, periodically gathers measurements and sends set-point corrections to the controlled devices. Having a wider view of the system, it is able to coordinate the efforts of multiple DGUs to yield an improved voltage profile and a better share of efforts among various DGUs.

For voltage correction purposes, it is attractive to combine the speed of local controllers with the coordination capabilities of a centralized controller. However, as mentioned in the Introduction, practical constraints may prevent the deployment of a communication system involving all DGUs. This leads to considering the hybrid control architecture of Fig. 1. In the latter, a subset of DGUs are under Combined Local and Centralized (CLC) control while the remaining are under local control only. This requires the centralized controller to take into account the (faster) reaction of all local controllers, including those on which it cannot act.

\section{LOWER-LEVEL: LOCAL CONTROL}

This section recalls the local voltage control used in [5].

\section{A. Reactive power control of a $D G U$}

In steady state the reactive power output of a DGU under local control follows the piecewise linear $V Q$ characteristic shown in Fig. 2. Such characteristic was proposed for instance in [9]. As long as the measured terminal voltage lies within the dead-band $\left[V_{\min 1}^{l o c}, V_{\max 1}^{l o c}\right]$, the produced reactive power $Q_{g}$ is kept at zero, which is usually preferred to minimize DGU internal losses. Outside the above mentioned dead-band, the DGU reacts to over- or under-voltage by consuming or producing reactive power, respectively. It is locked at its maximum reactive power production $Q_{\max }$ (resp. consumption $Q_{\min }$ ), if the voltage stays below $V_{\min 2}^{\text {loc }}$ (resp. above $V_{\max 2}^{l o c}$ ).

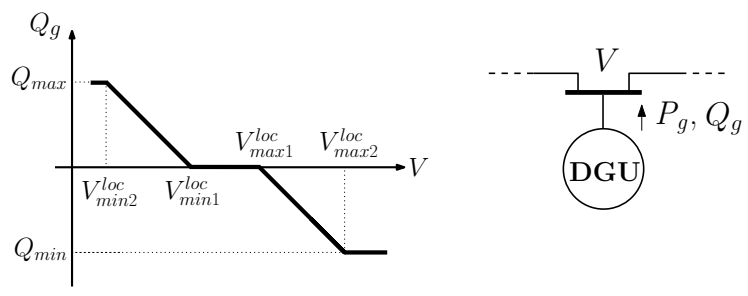

Fig. 2. Local level: steady-state $V Q$ characteristic [5]

A generic model of reactive power control, to be used for DGUs in local control only, is shown in Fig. 3.a. The terminal voltage $V$ and the generated reactive power $Q_{g}$ are measured, with the corresponding time constants $T_{V}$ and $T_{Q}$. The desired reactive power output $Q_{d}$ is given by the $V Q$ characteristic using the measured voltage. The difference between $Q_{d}$ and the measured reactive power is processed by a ProportionalIntegral controller. The output $V_{r e f}$ is the terminal voltage reference in a power-electronics based DGU, or the field voltage in a synchronous generator.

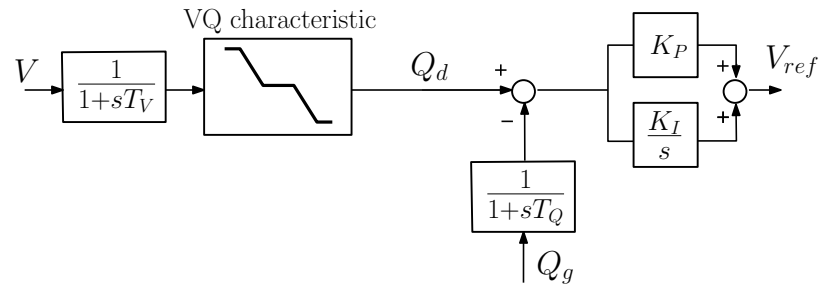

a: no interaction with the centralized controller

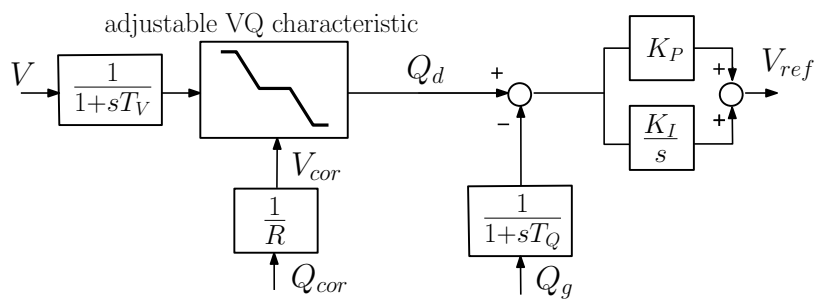

b: interaction with the centralized controller

Fig. 3. Local level: generic model of DGU reactive power control [5]

The "VQ characteristic" block corresponds to the piecewise linear variation of Fig. 2, which can be detailed as follows:

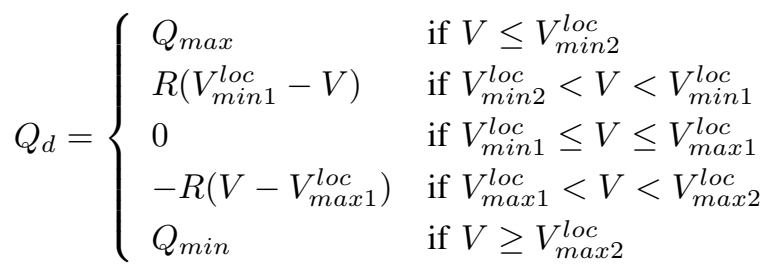

where the various voltage thresholds are defined in Fig. 2, and the same droop $R$ of the $V Q$ characteristic has been considered for under- and over-voltage, for simplicity.

The corresponding controller of a DGU under CLC control has an additional input for interaction with the upper level, as shown in Fig. 3.b. Namely, the local controller takes into account a reactive power correction $Q_{c o r}$ updated and sent at discrete times by the centralized controller, with the effect of shifting the $V Q$ characteristic, as detailed in the next section.

The change of reactive power output of one DGU affects the voltages at other buses, including DGU buses. Assuming that these DGUs are not operating in the dead-band of their $V Q$ characteristics, they will react to the voltage change by also adjusting their reactive powers. The interactions between locally controlled DGUs are such that the reactive power increase of a DGU causes reactive power decreases of other DGUs. The response time is at most a couple of seconds. 


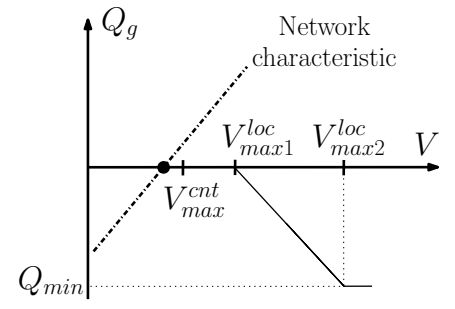

a. Initial operating point b. Overvoltage partly corrected by local control
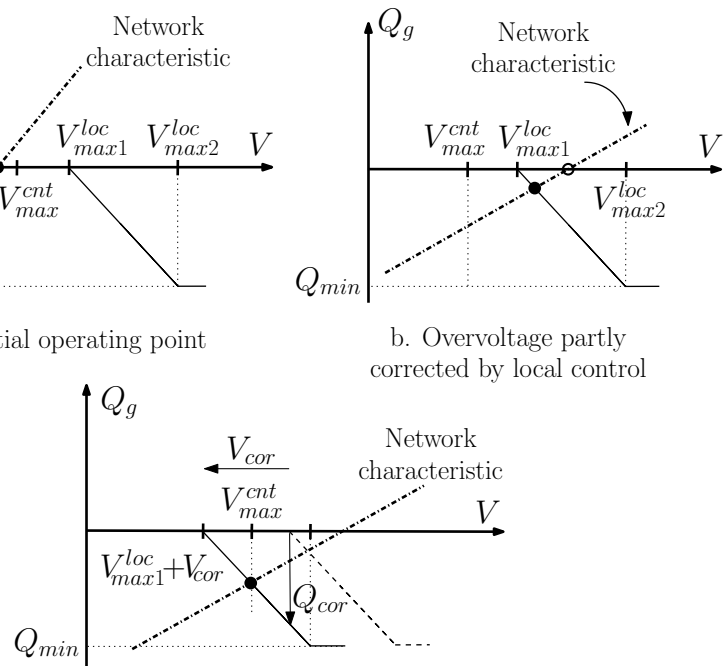

c. Final operating point after centralized control

Fig. 4. Example of over-voltage correction by local and centralized controls on adjustable $V Q$ characteristic (only high voltage part is shown) [5]

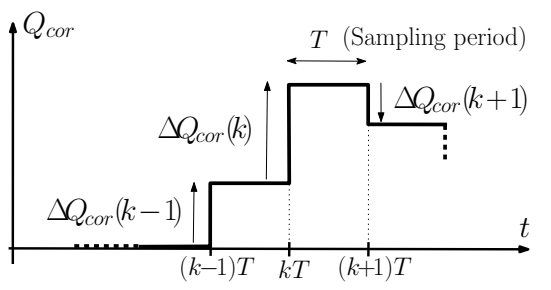

Fig. 5. Cumulation of reactive power corrections received from the centralized controller [5]

\section{B. Correction from the centralized controller}

Consider a DGU under CLC control. The correction $Q_{\text {cor }}$ received from the centralized controller causes a shift of the $V Q$ characteristic parallel to the $V$ axis. The purpose of this shift is explained with the following example.

Figure 4 shows an over-voltage situation and the subsequent actions to remove the violation. The initial operating point of the DGU, shown with a black dot, is at the intersection of the DGU and network $V Q$ characteristics. In the example of Fig. 4.a, the voltage lies in the dead-band. Thus, initially, the DGU operates at unity power factor. Let us assume that under the effect of a disturbance, the network characteristic changes and the DGU terminal voltage exceeds the upper limit $V_{\max 1}^{l o c}$. The circle in Fig. 4.b shows the situation with no reaction from the DGU, while the black dot is the result of the local control. The violation is partly (and promptly) corrected but the voltage is still above the upper voltage limit $V_{\max }^{c n t}$ monitored by the centralized controller.

The latter computes a sequence of corrections $\Delta Q_{c o r}$ and sends them to the local controller. The successively received corrections are cumulated as shown in Fig. 5, where $k$ is the discrete time, $T$ the sampling period and $Q_{c o r}$ the cumulated (or discrete integral) correction. The latter is used to shift the $V Q$ characteristic as shown in Fig. 4.c. Assuming operation on the sloping part of the $V Q$ characteristic, the voltage shift $V_{\text {cor }}$ corresponding to a given value of $Q_{\text {cor }}$ is:

$$
V_{\text {cor }}=\frac{Q_{\text {cor }}}{R}
$$

The upper level keeps on sending $\Delta Q_{\text {cor }}$ corrections until the voltage is brought at the $V_{\max }^{c n t}$ limit, as illustrated by the black dot in Fig. 4.c.

Note that the cumulated correction $Q_{c o r}$ is different from the DGU effective reactive power change, as seen from Fig. 4.c. A linear relation between both is derived in Section IV-B. The difference will be shown among the results of Section V-D.

Note also that different voltage limits are specified in the local and centralized controls. Local control aims at mitigating the voltage excursion in the very first seconds after a disturbance. The centralized control acts only if the resulting voltage exceeds the limit $V_{\max }^{c n t}$. This is the case if $V_{\max }^{c n t}<V_{\max 1}^{l o c}$.

A similar behaviour is obtained in case of under-voltage.

With the above correction, the expression of the "adjustable $V Q$ characteristic" block in Fig. 3.b can be detailed as follows:

$$
\begin{aligned}
& Q_{d}= \\
& \begin{cases}Q_{\max } & \text { if } V-V_{c o r} \leq V_{\min 2}^{l o c} \\
R\left(V_{\min 1}^{l o c}+V_{c o r}-V\right) & \text { if } V_{\min 2}^{\text {loc }}<V-V_{c o r}<V_{\min 1}^{l o c} \\
0 & \text { if } V_{\min 1}^{\operatorname{loc}} \leq V-V_{c o r} \leq V_{\max 1}^{l o c} \\
-R\left(V-V_{\max 1}^{l o c}-V_{c o r}\right) & \text { if } V_{\max 1}^{l o c}<V-V_{c o r}<V_{\max 2}^{l o c} \\
Q_{\min } & \text { if } V-V_{c o r} \geq V_{\max 2}^{l o c}\end{cases}
\end{aligned}
$$

\section{UPPER LEVEL: CENTRALIZED CONTROL}

This section summarizes the centralized control proposed in [5], adapted to act on the subset of DGUs under CLC control.

\section{A. MPC formulation}

A multi-time step optimization is at the heart of the MPC used at the centralized level.

The control variables are the corrections $\Delta Q_{\text {cor }}$ sent to the DGUs under CLC control.

At a discrete time $k$, the objective is to minimize over the next $N_{c}$ steps the deviations of the reactive powers of all DGUs, $\boldsymbol{Q}_{g}(k+i)$, from their last measured values, $\boldsymbol{Q}_{g}^{m}(k)$ :

$$
\min _{\boldsymbol{Q}_{g}, \boldsymbol{V}, \boldsymbol{\varepsilon}, \Delta \boldsymbol{Q}_{g}, \Delta \boldsymbol{Q}_{c o r}} \sum_{i=0}^{N_{c}-1}\left\|\Delta \boldsymbol{Q}_{g}(k+i)\right\|_{\boldsymbol{W}}^{2}+\|\varepsilon\|_{\boldsymbol{S}}^{2}
$$

where $\left(i=0, \ldots, N_{c}-1\right)$ :

$$
\Delta \boldsymbol{Q}_{g}(k+i)=\boldsymbol{Q}_{g}(k+i)-\boldsymbol{Q}_{g}^{m}(k)
$$

The second term in (4) involves the slack variables $\varepsilon$ aimed at relaxing the inequality constraints in case of infeasibility. $\boldsymbol{W}$ is a diagonal matrix allowing to give different weights to different DGUs. Matrix $\boldsymbol{S}$ is also diagonal with large entries to force the constraints.

The minimization is subject to the linearized relation between $\Delta \boldsymbol{Q}_{g}$ and the control variables $\left(i=0, \ldots, N_{c}-1\right)$ :

$$
\Delta \boldsymbol{Q}_{g}(k+i)=\boldsymbol{S}_{Q}^{\prime} \Delta \boldsymbol{Q}_{\text {cor }}(k+i)
$$


as well as the linearized predicted evolution of voltages over the future $N_{p}$ steps $\left(i=1, \ldots, N_{p}\right)$ :

$$
\boldsymbol{V}(k+i)=\boldsymbol{V}^{m}(k)+\boldsymbol{S}_{V} \Delta \boldsymbol{Q}_{g}(k+i-1)
$$

where $\boldsymbol{V}(k+i)$ is the vector of predicted bus voltages at time $k+i$. Equation (7) is initialized by the last received measurements $\boldsymbol{V}^{m}(k)$. $\boldsymbol{S}_{Q}^{\prime}$ and $\boldsymbol{S}_{V}$ are sensitivity matrices whose derivation is detailed in the next section. Matrix $S_{Q}^{\prime}$ relates the reactive powers of all DGUs to the corrections imposed to DGUs under CLC control. Thus, it is not a square matrix as in [5].

Finally, the following inequality constraints are imposed:

$$
\varepsilon=\left[\begin{array}{ll}
\varepsilon_{1} & \varepsilon_{2}
\end{array}\right]^{T} \geq \mathbf{0}
$$

$$
\begin{aligned}
& \text { for } i=1, \ldots, N_{p} \text { : } \\
& \left(-\varepsilon_{1}+V_{\min }^{c n t}\right) \mathbf{1} \leq \boldsymbol{V}(k+i) \leq\left(V_{\max }^{c n t}+\varepsilon_{2}\right) \mathbf{1} \\
& \text { for } i=0, \ldots, N_{c}-1 \text { : } \\
& \boldsymbol{Q}_{g}^{\min }(k) \leq \boldsymbol{Q}_{g}(k+i) \leq \boldsymbol{Q}_{g}^{\max }(k) \\
& \Delta \boldsymbol{Q}_{g}^{\text {min }} \leq \boldsymbol{Q}_{g}(k+i)-\boldsymbol{Q}_{g}(k+i-1) \leq \Delta \boldsymbol{Q}_{g}^{\max }
\end{aligned}
$$

where 1 denotes a unit vector, $\boldsymbol{Q}_{g}^{\min }, \boldsymbol{Q}_{g}^{\max }, \Delta \boldsymbol{Q}_{g}^{\min }$ and $\Delta \boldsymbol{Q}_{g}^{\max }$ are the lower and upper limits on DGU reactive powers and on their rates of change. The bounds $\boldsymbol{Q}_{g}^{\min }(k)$ and $\boldsymbol{Q}_{g}^{\max }(k)$ are updated at each time step based on the measured active power and terminal voltage, in accordance with the DGU capability curves [10]. In the constraints (11), $\boldsymbol{Q}_{g}(k-1)$ is set to $\boldsymbol{Q}_{g}^{m}(k)$.

\section{B. Derivation of sensitivity matrices}

The sensitivity matrix $\boldsymbol{S}_{V}$ (resp. $\boldsymbol{S}_{Q}^{\prime}$ ) expresses by how much the bus voltages (resp. the DGU reactive powers) vary following a small change $\Delta \boldsymbol{Q}_{\text {cor }}$ of the control variables.

A graphic view is given in Fig. 6, showing how a variation of $\Delta Q_{\text {cor }}$ modifies the $V Q$ characteristic in a DGU under CLC control. Assuming operation on the inclined part of the $V Q$ characteristic, the figure shows the resulting changes of voltage $\Delta V$ and reactive power $\Delta Q_{g}$.

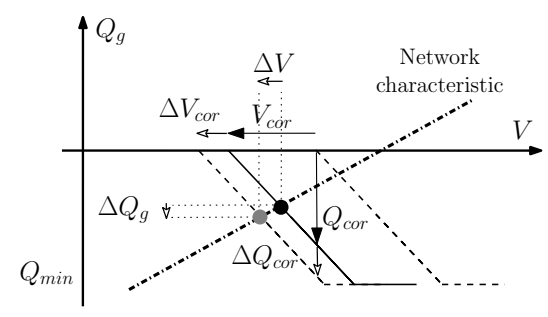

Fig. 6. Graphic representation of the effects of a small control change $\Delta Q_{\text {cor }}$ on a DGU under CLC control [5]

The equation of the solid black line is:

$$
Q_{g}-Q_{c o r}=-R\left(V-V_{\max 1}^{l o c}\right)
$$

involving the already defined droop $R$. Considering small deviations denoted with $\Delta$, Eq. (12) gives:

$$
\Delta Q_{g}-\Delta Q_{\text {cor }}=-R \Delta V
$$

The corresponding equation for a DGU under local control only can be easily derived by setting $\Delta Q_{\text {cor }}$ to zero in (13).

The whole set of equations can be written in compact matrix form as:

$$
\Delta \boldsymbol{Q}_{g}=\left[\begin{array}{c}
\mathbf{0} \\
\Delta \boldsymbol{Q}_{c o r}
\end{array}\right]-\boldsymbol{R} \Delta \boldsymbol{V}
$$

where the size of the zero vector $\mathbf{0}$ is the number of DGUs under local control only, and $\boldsymbol{R}$ is a diagonal matrix whose diagonal elements are the various droop coefficients.

The variation of the bus voltages with the DGU reactive powers is given by:

$$
\Delta \boldsymbol{V}=\boldsymbol{S}_{V} \Delta \boldsymbol{Q}_{g}
$$

where $\boldsymbol{S}_{V}$ can be obtained from the transposed inverse of the power flow Jacobian matrix. Alternatively, each column of the matrix can be computed by running a power flow calculation with one DGU reactive power slightly modified, and dividing the bus voltage variations by the reactive power variation considered (see e.g. [11] for more details).

By substituting (15) in (14), one easily obtains:

$$
\Delta \boldsymbol{Q}_{g}=\left(\boldsymbol{I}+\boldsymbol{R} \boldsymbol{S}_{V}\right)^{-1}\left[\begin{array}{c}
\mathbf{0} \\
\Delta \boldsymbol{Q}_{c o r}
\end{array}\right]
$$

where $\boldsymbol{I}$ is a unit matrix. This equation can be rewritten as:

$$
\Delta \boldsymbol{Q}_{g}=\boldsymbol{S}_{Q}^{\prime} \Delta \boldsymbol{Q}_{\text {cor }}
$$

where $\boldsymbol{S}_{Q}^{\prime}$ is obtained from $\left(\boldsymbol{I}+\boldsymbol{R} \boldsymbol{S}_{V}\right)^{-1}$ by removing the columns relative to DGUs under local control only.

The above derivation assumes that all DGUs operate on the sloping portion of their $V Q$ characteristics. This approximation is discussed in [5], where it is shown that the inaccuracy can be compensated by the MPC scheme.

\section{Simulation Results}

\section{A. Test system}

The performance of the proposed controls are illustrated on the 75-bus, $11-\mathrm{kV}$ network shown in Fig. 7 [12]. It is connected through a $33 / 11 \mathrm{kV}$ transformer to the external grid, represented by a Thévenin equivalent.

The network feeds 52 loads, of which 38 are modelled as constant current (resp. impedance) for active (resp. reactive) power, and 14 with equivalent (small) induction motors. Note that all loads are treated as constant powers when computing the sensitivity matrices involved in the MPC.

The network hosts 22 DGUs, consisting of 3.33-MVA doubly fed induction generators driven by 3-MW wind-turbines, and 3-MVA synchronous generators driven by 2.55-MW turbines, respectively. The DGU models and parameters were taken from [13], [14], and simplified in accordance with the dynamics of interest in this work. The DGU capability curves are such that the units can operate with a power factor between 0.9 and 1.0 in both under- and over-excited modes. 


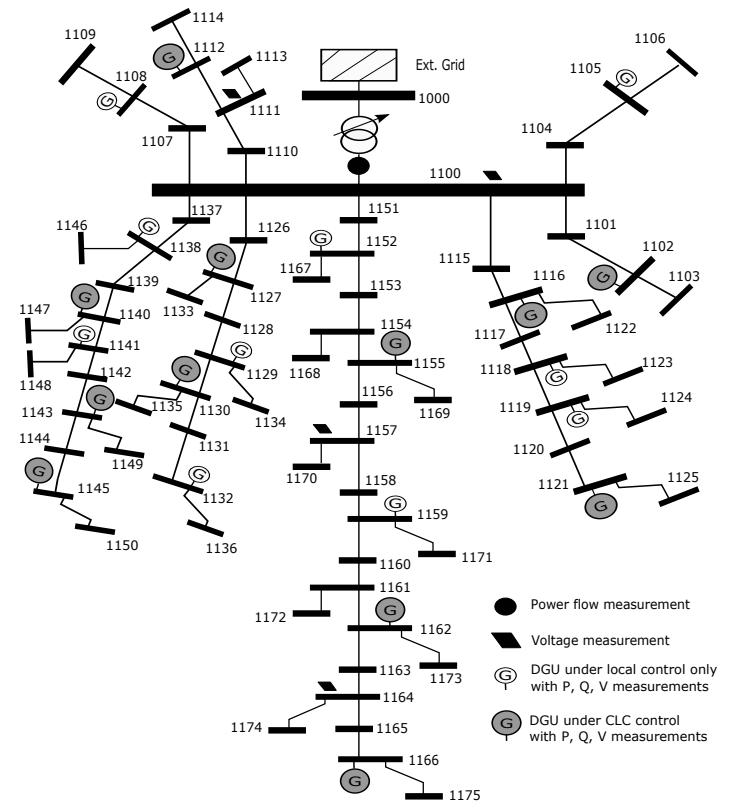

Fig. 7. 75-bus test system

\section{B. Control settings}

Ten DGUs are assumed to be under local control only. The remaining twelve, under CLC control, are spread over different feeders, as shown by the gray filled disks in Fig. 7.

The terminal voltages and the active/reactive powers of the 22 DGUs are measured, as well as the voltages at four nonDGU buses. The measurements are received every $10 \mathrm{~s}$ by the centralized controller, which sends the corrections $\Delta \boldsymbol{Q}_{\text {cor }}$ at the same rate. The units respond to these corrections within a few seconds, due to their internal dynamics.

The following voltage thresholds and limits were used in all simulations: $V_{\max 2}^{\text {loc }}=1.08 \mathrm{pu}, V_{\max }^{c n t}=V_{\max 1}^{\text {loc }}=1.03 \mathrm{pu}$, $V_{m i n}^{c n t}=V_{\min 1}^{l o c}=0.97 \mathrm{pu}$ and $V_{\min 2}^{l o c}=0.92 \mathrm{pu}$.

In the objective function (4), $\boldsymbol{W}$ has been set to a unit matrix and the diagonal entries of $\boldsymbol{S}$ to $10^{4}$, where all voltages and slack variables are in per unit on the network voltage base.

Since distribution transformers are usually equipped with automatic Load Tap Changers (LTCs), voltages can be also controlled by adjusting the LTC voltage set-point. However, increasing the number of tap changes reduces the LTC lifetime, and considering the higher accuracy and speed offered by power electronics-based DGUs, the latter are likely to be preferred to LTCs in future active distribution networks. Hence, in this work, voltage correction is performed without the contribution of the LTC, although the latter can be easily incorporated as explained in [4].

\section{Simulation tool}

Simulations were performed with RAMSES, a dynamic simulation software developed at the Univ. of Liège [15]. The local control of Fig. 3 is embedded in the differential-algebraic model of the DGU unit, while the centralized controller acts at discrete times only. The quadratic programming problem (411) is solved with the VE17AD package from Harwell [16].

\section{Illustrative example}

All voltages are initially within the $[0.971 .03]$ pu deadband common to both control levels, and all DGUs operate with a unity power factor.

The simulation results deal with the response to a $0.05 \mathrm{pu}$ drop of the external grid voltage at $t=10 \mathrm{~s}$.

Figure 8 shows the voltage evolutions at a sample of buses throughout the grid. The voltage drop at $t=10 \mathrm{~s}$ is large enough to move the operating point outside the dead-band of many $V Q$ characteristics (see Fig. 2). Therefore, the DGUs whose terminal voltage drops below $V_{\min 1}^{l o c}=0.97 \mathrm{pu}$ start producing reactive power right after the disturbance. The other DGUs keep operating at unity power factor. The voltages are partly but rapidly corrected, leading to fewer buses in low voltage situation.

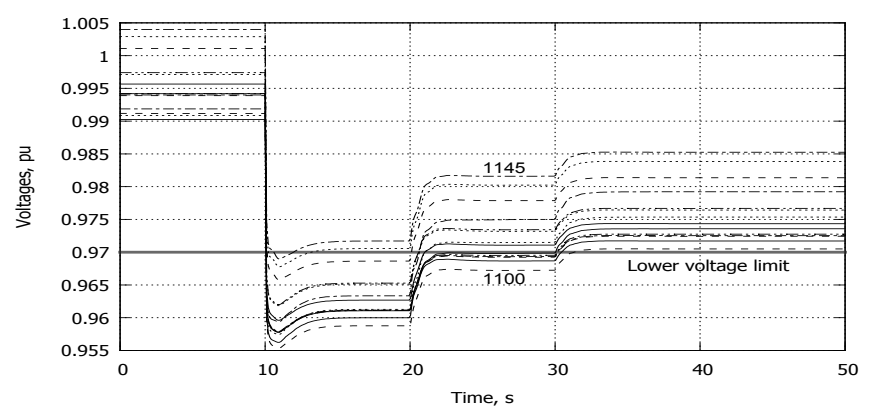

Fig. 8. Voltages at a sample of MV buses

At the next discrete time step, $t=20 \mathrm{~s}$, the centralized controller, which has sensed the unsatisfactory voltages, starts sending corrections. The latter shift the VQ characteristics of the 12 DGUs under CLC control, leading them to increase their reactive power productions. It succeeds bringing all voltages within the desired limits in two discrete steps (at $t=20$ and $30 \mathrm{~s}$, respectively).

Figure 9 shows how DGUs interact. As the centralized controller increases the reactive power of the DGUs under its control (e.g. see DGU at bus 1155 or 1162), the network voltages increase and the DGUs under local control only have their reactive power decreased (e.g. see DGU at bus 1152) and return to unity power factor at $t \simeq 35 \mathrm{~s}$.

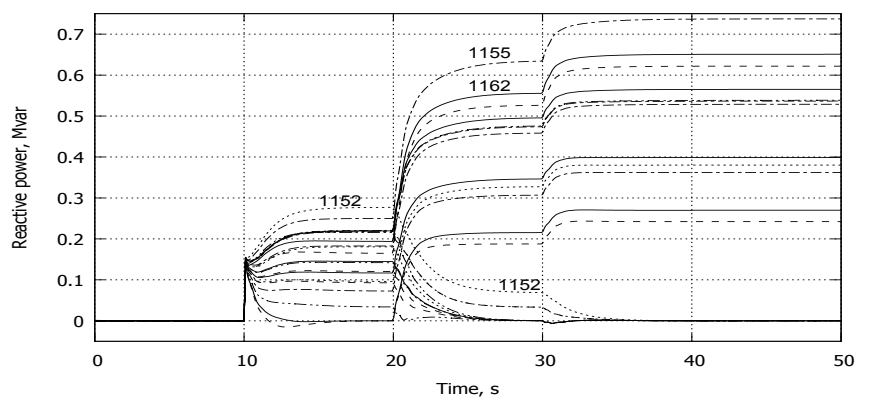

Fig. 9. Reactive power produced by a sample of DGUs

In other words, DGUs under local control only participate in the initial correction of the voltage violation, and inject 
reactive power for a few tens of seconds only. The centralized controller anticipates this behaviour (through its sensitivity matrices) and properly adjust the DGUs under its control.

Figure 10, focusing on the DGU at bus 1155, shows the cumulated correction $Q_{c o r}$ (dashed line), received from the centralized controller, and the resulting reactive power production $Q_{g}$ (solid line). The former is increased in two steps, and the latter follows according to the DGU dynamics. In the final steady state, the two curves differ since: (i) a correction $\Delta Q_{c o r}$ applied to a DGU does not yield an equal variation $\Delta Q_{g}$ of the that DGU, as shown graphically in Fig. 4; (ii) the reactive power of a DGU is impacted by the corrections applied to the other DGUs. This last effect is captured by the off-diagonal elements of matrix $\boldsymbol{S}_{Q}^{\prime}$, which show that the DGUs with the largest impact are those connected to buses 1162 and 1166.

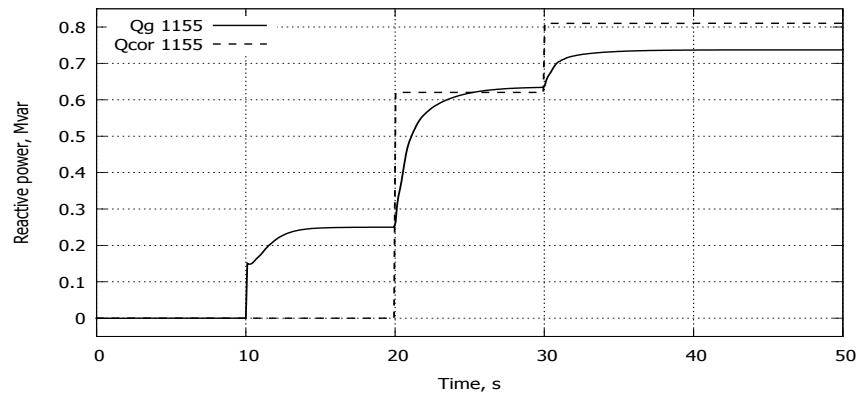

Fig. 10. DGU at bus 1155: cumulated correction vs. reactive power production

Figure 11 shows the reactive power and the voltage of the DGU at bus 1152. Since this unit is under local control only, it does not produce reactive power as long as the voltage lies in between $V_{\min 1}^{l o c}=0.97$ and $V_{\max 1}^{l o c}=1.03 \mathrm{pu}$. In response to the initial disturbance, the voltage drops below $0.97 \mathrm{pu}$ and the unit increases its reactive power. The time taken to reach the maximum, around $5 \mathrm{~s}$, is due to the measurement time constant, the PI controller (see Fig. 3), and other delays in the DGU excitation system. This response time is still short with respect to the $10 \mathrm{~s}$ sampling time of the centralized controller, which allows using a static, sensitivity-based moded in the MPC formulation.

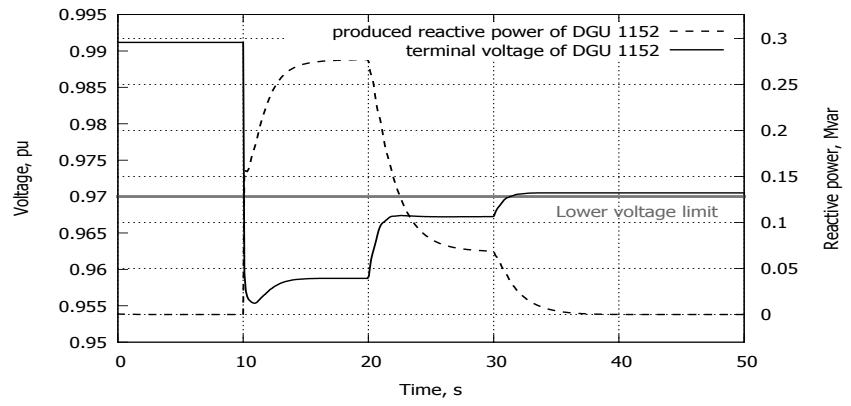

Fig. 11. DGU at bus 1152: terminal voltage and reactive power

\section{CONCLUSION}

In this paper a hybrid control architecture has been presented for corrective control of voltages in active distribution networks. This scheme is of interest when all DGUs cannot be controlled centrally. It includes local controllers in the DGUs, and a centralized one steering only a subset of units. Each local controller relies on a piecewise linear VQ characteristic and uses its voltage measurement to promptly react to disturbances. The discrete-time centralized controller computes power corrections whose effect is to adjust the local $V Q$ characteristics.

The simulation results show the fast but partial correction by the local controllers, followed by the smooth, coordinated control of a subset of DGUs. If the voltage limits specified in the centralized control correspond to the dead-band of the $V Q$ characteristics, the whole reactive power effort is eventually transfered to the centrally controlled DGUs while the DGUs under local control only return to unity power factor operation.

\section{REFERENCES}

[1] V. A. Evangelopoulos, P. S. Georgilakis, and N. D. Hatziargyriou, "Optimal operation of smart distribution networks: A review of models, methods and future research," Electric Power Systems Research, vol. 140, pp. 95-106, 2016.

[2] P. Kotsampopoulos, N. Hatziargyriou, B. Bletterie, and G. Lauss, "Review, analysis and recommendations on recent guidelines for the provision of ancillary services by distributed generation," Proc. IEEE Intern. Workshop on Intelligent Energy Systems, pp. 185-190, 2013.

[3] A. Seack, J. Kays, and C. Rehtanz, "Time series based distribution grid planning approach with decentralised voltage regulation," Proc. Power Systems Computation Conference, Wroclaw, Aug. 2014.

[4] G. Valverde and T. Van Cutsem, "Model predictive control of voltages in active distribution networks," IEEE Trans. on Smart Grid, vol. 4, pp. 2152-2161, Dec. 2013.

[5] H. Soleimani Bidgoli and T. Van Cutsem, "Combined local and centralized voltage control in active distribution networks," unpublished, submitted for publication in IEEE Trans. on Power Systems (under second review).

[6] Y. Chistyakov, E. Kholodova, K. Netreba, A. Szabo, and M. Metzger, "Combined central and local control of reactive power in electrical grids with distributed generation," Proc. IEEE ENERGYCON Conference, pp. 325-330, Sep. 2012.

[7] N. Jayasekara, P. Wolfs, and M. A.S. Masoum, "An optimal management strategy for distributed storages in distribution networks with high penetrations of PV," Electric Power Systems Research, vol. 116, pp. 147-157, 2014.

[8] M. Farina, A. Guagliardi, F. Mariani, C. Sandroni, and R. Scattolini, "Model predictive control of voltage profiles in MV networks with distributed generation," Control Eng. Practice, vol. 34, pp. 18-29, 2015.

[9] T. Kerber, G. Kerber, M. Werner, M. Finkel, and M. Wiest, "Decentralized voltage control in $20 \mathrm{kV}$ distribution grids by controlling reactive power of inverters," Proc. CIRED Workshop, no. 0112, 2014.

[10] G. Valverde and J. J. Orozco, "Reactive power limits in distributed generators from generic capability curves," Proc. IEEE PES General Meeting, Washington DC., Jul. 2014.

[11] H. Soleimani Bidgoli, M. Glavic, and T. Van Cutsem, "Receding-horizon control of distributed generation to correct voltage or thermal violations and track desired schedules," Proc. Power Systems Computation Conference, Genoa, Jun. 2016.

[12] "United Kingdom generic distribution network (UKGDS)," Available: http://sedg.ac.uk.

[13] J. D. Hurley, L. N. Bize, and C. R. Mummert, "The adverse effects of excitation system var and power factor controllers," IEEE Trans. on Energy Conversion, vol. 14, pp. 1636-1645, 1999.

[14] G. Tsourakis, B. M. Nomikos, and C. D. Vournas, "Effect of wind parks with doubly fed asynchronous generators on small-signal stability," Electric Power Systems Research, vol. 79, pp. 190-200, 2009.

[15] P. Aristidou, S. Lebeau, and T. Van Cutsem, "Power system dynamic simulations using a parallel two-level Schur-complement decomposition," IEEE Trans. on Power Systems, vol. 31, pp. 3984-3995, 2016.

[16] "HSL. a collection of fortran codes for large scale scientific computation," Available: http://www.hsl.rl.ac.uk. 